\title{
Médiévales
}

Langues, Textes, Histoire

57 | automne 2009

Langages politiques, $\mathrm{XII}{ }^{\mathrm{e}}-\mathrm{XV}^{\mathrm{e}}$ siècle

\section{Les harangues de la bataille de l'Étendard (1138)}

The harangues of the Battle of the Standard (1138)

\section{Xavier Storelli}

\section{OpenEdition}

Journals

Édition électronique

URL : https://journals.openedition.org/medievales/5802

DOI : 10.4000/medievales.5802

ISSN : 1777-5892

\section{Éditeur}

Presses universitaires de Vincennes

\section{Édition imprimée}

Date de publication : 20 décembre 2009

Pagination : 15-32

ISBN : 978-2-84292-241-2

ISSN : 0751-2708

Référence électronique

Xavier Storelli, «Les harangues de la bataille de l'Étendard (1138) », Médiévales [En ligne], 57 | automne 2009, mis en ligne le 18 janvier 2012, consulté le 22 avril 2022. URL : http://journals.openedition.org/ medievales/5802 ; DOI : https://doi.org/10.4000/medievales.5802 
Médiévales 57, automne 2009, p. 15-32

Xavier STORELLI

LES HARANGUES DE LA BATAILLE DE L'ÉTENDARD (1138)

Par un matin de brume et d'angoisse, le 22 août 1138, Gautier Espec, seigneur d'Helmsley et de Kirkham, s'adressant aux troupes anglo-normandes juste avant la bataille de l'Étendard, légitime son allocution par sa connaissance du passé, qui lui permettrait de saisir, mieux que ses compagnons, la portée de l'affrontement imminent, d'en prévoir l'issue et les conséquences. Ælred de Rievaulx, qui fait discourir ce noble personnage, fondateur de son monastère, dans sa Relatio de Standardo, élabore une mise en abyme, un jeu de miroir : la composition rhétorique qui orne la narration historiographique devient ici une défense et une illustration de l'histoire elle-même ${ }^{1}$.

Les chroniqueurs anglo-normands $\mathrm{du} \mathrm{XI}^{\mathrm{e}}$, et surtout $\mathrm{du} \mathrm{XII}{ }^{\mathrm{e}}$ siècle, accordent une place singulière aux harangues militaires dans leurs récits ${ }^{2}$. Ces discours sont censés avoir été prononcés afin de galvaniser les énergies, au tout début d'une expédition ou bien juste avant le choc. Ce sont des compositions littéraires qui permettent, après coup, d'agrémenter la description d'une confrontation guerrière, souvent d'importance, et d'en éclairer le sens.

Ces harangues occupent une position originale dans le récit et présentent un double intérêt. D'une part, elles constituent un élément clef de la construction du discours historiographique sur les batailles : le chroniqueur prête sa plume et son inspiration à un chef de guerre sans confondre totalement sa voix avec la sienne. À ce titre, elles introduisent une certaine complexité dans la caractérisation des acteurs et l'organisation de la matière événementielle ${ }^{3}$. D'autre part, en combinant toute une gamme de motifs rhé-

1. Ælred de Rievaulx, Relatio de Standardo, Chronicles of the Reigns of Stephen, Henry II and Richard I, R. HowletT éd., t. 3, Londres, 1886, p. 185.

2. J. BliESE, « The Courage of the Normans. A Comparative Study of Battle Rhetoric », Nottingham Medieval Studies, t. 35, 1991, p. 1-26.

3. M. AURELl, «Rapport introductif » et X. STORELLI, « Convaincre pour vaincre : place et fonction des harangues militaires dans l'historiographie anglo-normande $\left(\mathrm{XI}^{\mathrm{e}} \mathrm{s}\right.$.-début du XIII ${ }^{\mathrm{e}}$ s.) », dans M. AURELl dir., Convaincre et persuader : communication et propagande aux $\mathrm{XII}^{e}$ et XIII ${ }^{e}$ siècles, Poitiers, 2007, p. 40-43 et p. 53-80. 
toriques, elles constituent une source d'information sur les motivations des combattants, sur la représentation d'une parole d'autorité qui peut conduire des hommes à consentir au sacrifice suprême ${ }^{4}$.

C'est sur ce deuxième aspect que se concentre le présent travail, à partir du cas bien particulier de la bataille de l'Étendard, livrée au début de la guerre civile (1138-1153) qui opposa, en Angleterre et en Normandie, le roi Étienne de Blois, neveu de Henri $\mathrm{I}^{\mathrm{er}}$ Beauclerc, à la fille de ce dernier, l'impératrice Mathilde, épouse en secondes noces de Geoffroy Plantagenêt.

Dès le couronnement d'Étienne (décembre 1135), le roi David I ${ }^{\text {er }}$ d'Écosse défend la cause de Mathilde. Entrecoupées de trêves et de négociations, les hostilités renaissent sans cesse car le roi d'Écosse rêve de restaurer, à son profit, un comté de Northumbrie dont la création serait inacceptable pour le souverain d'Angleterre. En avril 1138, David se lance dans une nouvelle expédition. À la fin du mois de juillet, il entrâne vers le sud une armée imposante et disparate (composée de Pictes de Galloway, d'hommes des îles et de Moray, de combattants saxons de Northumbrie et de chevaliers normands) ; il franchit les rivières Tyne et Tees, vers York, afin, dit-on, de ravager l'Angleterre ${ }^{5}$.

Le mardi 22 août 1138, non loin de Northallerton (3 à 4 kilomètres à l'est), à Cowton Moor, une armée fidèle à Étienne de Blois (mais en son absence) vainc les troupes écossaises. Les Anglo-Normands ont adopté un dispositif défensif : les chevaliers combattent à pied, aux côtés des archers. L'assaut des guerriers de Galloway se brise sur les lignes de fer adverses et les volées de flèches. L'armée du roi David et de son fils, Henri, est taillée en pièces ${ }^{6}$. Les barons du Yorkshire ne cherchent pourtant pas à exploiter

4. J. BliESE, «Rhetoric and Morale : a Study of Battle Orations from the Central Middle Ages », Journal of Medieval History, t. 15, 1989, p. 201-206 ; " The Just War as Concept and Motive in the Central Middle Ages ", Medievalia et Humanistica. Studies in Medieval and Renaissance Culture, New Series, t. 17, 1991, p. 1-26. Voir aussi S. BAGGE, «Oratory and Politics in the Sagas », dans J.-Ph. GENET dir., L'Histoire et les nouveaux publics dans l'Europe médiévale XIII ${ }^{e}-X V^{e}$ siècles. Actes du colloque international organisé par la Fondation Européenne de la Science à la Casa de Velázquez, Madrid, 23-24 avril 1993, Paris, 1997, p. 215-228. Sur la question du consentement au pouvoir, voir F. FORONDA, «La propagande monarchique dans la Castille du XIII ${ }^{\mathrm{e}}$ siècle. Considérations autour du Libro de los doze sabios », dans M. AuRell dir., Convaincre et persuader..., p. 279-280.

5. La bibliographie sur la guerre civile du règne du roi Étienne est imposante, citons seulement: D. Crouch, The Reign of King Stephen, 1135-1154, Londres, New York, 2000 ; D. Matthew, King Stephen, Londres, New York, 2002 ; P. Dalton et G. J. White dir., King Stephen's Reign (1135-1154), Woodbridge, 2008. Sur les difficultés de l'été 1138 et la campagne de l'Étendard voir D. Crouch, The Reign of King Stephen..., chap. 4 et plus particulièrement p. 78-84 ; R. R. L. Graeme, The Normans in Scotland, Edinbourgh, 1954, p. 256-270.

6. Ælled de RievaulX, Relatio..., op. cit., p. 196-199; JeAn DE WorCester, Chronicon Ihoannis Wigornensis, P. McGuRK éd., t. 3, Oxford, 1998, p. 252-254; RICHARD D'HeXHAM, De gestis regis Stephani, Chronicles of the Reigns of Stephen, Henry II and Richard I, R. HOWLETT éd., t. 3, Londres, 1886, p. 162-165 ; JEAN D'HEXHAM, Symeonis historia regum continuata per Joh. Hagustaldensem, Symeonis monachi opera omnia, T. ARNold éd., Londres, t. 2, 1885, chap. 6, p. 293-295 ; Henri de Huntingdon, Historia Anglorum, D. Greenway éd., Oxford, 
leur victoire. David se contente d'assiéger le château de Carham : la tentative de «percée écossaise » a échoué ${ }^{7}$.

Ce n'est pas la bataille en elle-même qui nous intéresse ici, mais sa préparation, en particulier les différentes prises de paroles qui l'ont précédée. Si la plupart des chroniqueurs anglo-normands de l'époque mentionnent cette « victoire anglaise », cinq seulement font un récit détaillé de la campagne et de l'affrontement. Deux d'entre eux ont composé, moins de vingt ans après les faits, une exhortation militaire qui aurait été prononcée le jour même de la bataille : Henri de Huntingdon fait discourir l'évêque des Orcades, Raoul Novel (chassé à cette époque de son siège épiscopal et suppléant de l'influent et charismatique archevêque d'York, Toustain), et Ælred de Rievaulx «donne » la parole au baron Gautier Espec, fondateur de son monastère ${ }^{8}$.

Henri, archidiacre de Huntingdon n'écrivit son discours que deux ans après le succès anglo-normand ${ }^{9}$. Il inséra cette harangue dans l'histoire nationale synthétique et brillante, riche en anecdotes, qu'il entreprit à la demande de son puissant patron, l'évêque Alexandre de Lincoln ${ }^{10}$.

C'est plus tard, probablement vers 1155-1157, qu' Ælred, abbé de Rievaulx, rédigea un opuscule intitulé Relatio de Standardo ${ }^{11}$. Le discours prêté à Gautier Espec en représente à peu près le quart. Il s'agit sans doute d'une reprise des pages que Henri de Huntingdon consacra à l'événement car le passage qui concerne la bataille chez Henri et l'opuscule d'Ælred commencent d'une façon très semblable, comme si le moine cistercien avait souhaité compléter l'œuvre de l'archidiacre en reprenant la narration du combat et le discours qui le précède ${ }^{12}$. La connaissance que l'abbé de Rievaulx a des hommes et du contexte des opérations est bien plus fine que celle de Henri de Huntingdon puisque Ælred avait été élevé à la cour du roi David d'Écosse et qu'il était moine à Rievaulx - abbaye voisine de Northallerton - lors de la confrontation ${ }^{13}$. Pris entre son amitié pour le roi d'Écosse et son souci de défendre l'Église du Yorkshire, à la croisée de deux

1996, L. X, chap. 9, p. 716-718 ; J. Bradbury, Stephen and Matilda. The Civil War of 11391153, Stroud, 1996, p. 32-37.

7. Richard D'HeXham, De gestis..., op. cit., p. 165-166.

8. Henri de Huntingdon, Historia..., op. cit., L. X, chap. 9, p. 714-716. Ælered DE RiEvaulX, Relatio..., p. 185-189.

9. Henri De Huntingdon, Historia..., op. cit., p. LXX et LXXIII-LXXV.

10. Ibid., p. LII-LVIII.

11. Sur la nature de l'opuscule voir A. GLIDDEN, «Ælred the Historian: the Account of the Battle of the Standard », SMCH (Studies in Medieval Cistercian History), t. 11, 1987, p. 182183 ; D. BAKER, «Ailred of Rievaulx and Walter Espec », The Haskins Society Journal, t. 1, 1989, p. 98 ; A. GRANSDEN, Historical Writing in England, ca 550 to ca 1307, Londres, 1974, p. 213 ; E. FreEman, Narratives of a New Order. Cistercian Historical Writing in England, 1150-1220, Turnhout, 2002, p. 32-47.

12. A. SQuire, Elred of Rievaulx. A Study, Kalamazoo, 1981 (1969), p. 76 ; ÆLred DE RievaulX, Relatio..., op. cit., p. 181 ; HenRi De HunTingdon, Historia..., op. cit., L. X, chap. 7, p. 712 .

13. Gautier Daniel, Vita Aelredi, M. Powicke éd., Oxford, 1978 (1950), p. XCI. 
cultures anglaise et française, la vie d'Ælred reflète la tension entre des fidélités multiples qu'il résout dans sa création historiographique ${ }^{14}$.

Il est peu probable que les harangues militaires aient été déclamées sur les champs de bataille, du moins sous la forme que nous leur connaissons ; il y a même de fortes chances pour qu'elles n'aient jamais été prononcées du tout. Il est donc aisé de confronter les deux discours prêtés à Raoul Novel et à Gautier Espec et de conclure à l'inconséquence des chroniqueurs et à l'inconsistance de ces deux prétendues exhortations, puisque le même discours est attribué, mais sous des formes distinctes, à deux personnes différentes ${ }^{15} \ldots$ La seule réalité des ces compositions serait littéraire. Or, s'il est vrai que ces oraisons ne sont pas l'exacte retranscription de paroles prononcées telles quelles, leurs thématiques sont sans doute très proches des «slogans » employés pour rallier des soutiens au camp d'Étienne de Blois. Assez exceptionnellement, dans le cas de la bataille de l'Étendard, la voix des orateurs de chair paraît imprégner profondément celle des «tribuns » de parchemin ${ }^{16}$.

Pour bien comprendre la portée de ces discours, il faut se rappeler qu'ils ne sont pas indifférents au contexte politique et militaire dans lequel ils sont censés avoir été prononcés. John Bliese ne considère-t-il pas que la harangue prêtée à Gautier Espec reflète l'inquiétude profonde des troupes anglo-normande au moment de la campagne ? En effet, les barons avaient de quoi se faire du mauvais sang: le roi Étienne était absent alors que l'armée ennemie déferlait sur leurs terres et qu'ils craignaient des défections dans leurs rangs ${ }^{17}$.

Il faut, en outre, associer aux discours qui auraient été prononcés le jour même de la bataille les propos tenus, au début de l'expédition, par Toustain, l'archevêque d'York et rapportés par le seul Richard, chanoine puis prieur d'Hexham, dans son sobre et austère De gestis regis Stephani et de bello Standardii. Ces paroles donnent une profondeur temporelle et idéologique aux deux précédents discours ${ }^{18}$.

Il faut enfin se référer au travail de deux historiens qui complètent notre connaissance des faits sans proposer de morceaux rhétoriques : Jean de Worcester, moine bénédictin, qui poursuivit au début $\mathrm{du}_{\mathrm{XII}}^{\mathrm{e}}$ siècle, à la

14. D. WALKER, «Cultural Survival in an Age of Conquest », dans R. R. DAviES dir., Historical Essays Presented to Glanmor Williams, Cardiff, 1984, p. 44.

15. D. BAKER, «Ailred of Rievaulx... », op. cit., p. 95 ; A. GranSDEN, Historical Writing..., op. cit., p. 215.

16. J. BLIESE, « Elred of Rievaulx's Rhetoric and Morale at the Battle of the Standard, 1138 », Albion, t. 20, 4, 1988, p. 543-556 ; ID., « The Battle Rhetoric of Ælred of Rievaulx », The Haskins Society Journal, t. 1, 1989, p. 99-107.

17. ÆLRED DE RIEVAulX, Relatio..., op. cit., p. 185 ; J. Bliese, «Ælred of Rievaulx’s Rhetoric... », op. cit., p. 555-556; ID., «The Battle Rhetoric... », op. cit., p. 102.

18. Richard D'HeXham, De gestis..., op. cit., p. 160-161 ; A. Gransden, Historical Writing..., op. cit., p. 216-218. 
demande de l'évêque Wulfstan, une chronique universelle inspirée de la chronique anglo-saxonne et Jean, prieur d'Hexham vers 1160, qui rédigea sous le règne de Henri II une chronique couvrant une vingtaine d'années, de 1130 à 1153, continuation de l'Historia regum attribuée à Siméon de Durham ${ }^{19}$.

\section{Les textes et l'écriture}

Avant d'entrer plus avant dans l'argumentation de ces discours, il faut évoquer les conditions de leur rédaction et leur valeur historique.

La tradition de la rhétorique belliqueuse insérée dans le discours historiographique remonte à l'Antiquité : les écrivains classiques usèrent abondamment de ce procédé afin d'agrémenter leurs récits. On considère parfois que les discours des chroniques anglo-normandes ne seraient qu'ornementaux, simples plagiats des productions antiques. Or, rien n'est plus faux. S'il est avéré que ces compositions s'inspirent souvent, sur le plan stylistique, de modèles classiques, l'argumentaire qui s'y développe, à de rares exceptions près, est bien propre au monde médiéval et à la chrétienté occidentale. L'influence des modèles bibliques (notamment des harangues tirées du Livre des Maccabées) modifie le cadre conceptuel propre aux discours antiques. À l'argumentaire surtout patriotique, tactique et politique de l'art oratoire grec et romain s'ajoute l'attention portée à l'état spirituel des troupes, à leur comportement moral, à la légitimité de leur cause et aux desseins de Dieu, seul maître de l'histoire ${ }^{20}$.

Reste à se demander si ces trois discours ne correspondraient pas à une construction narrative venant habiller des faits de nature militaire et sociologique dans le seul but de magnifier, après coup, les exploits des nobles anglo-normands. En ce sens, ne sont-ils pas tous issus d'une même source? Ne seraient-ils que les reprises d'une interprétation historiographique initiale et fondatrice ? Cela fragiliserait la portée de ces témoignages qui seraient alors plus le reflet d'une élaboration littéraire que d'une réelle campagne de propagande.

La question mérite d'autant plus d'être posée qu'Ælred, on l'a dit, avait certainement choisi de réécrire la description de la bataille qu'en avait donnée Henri de Huntingdon. Une édition de l'Historia Anglorum était disponible, au XIII ${ }^{\mathrm{e}}$ siècle et peut-être avant, dans la bibliothèque de Rievaulx et les moines de Durham et de Kirkstall en possédaient une copie dès le $\mathrm{XII}^{\mathrm{e}}$ siècle $^{21}$.

19. A. Gransden, Historical Writing..., op. cit., p. 143-149, p. 261.

20. X. STORELLI, «Convaincre pour vaincre...», op. cit., p. 56-65.

21. Catalogue de l'abbaye de Rievaulx. Bibliotheca Aelrediana, Old Catalogues, Editions and Studies Concerning St. Alred of Rievaulx, Dom A. Hoste éd., Steenbrugis, 1962, p. 161, $\mathrm{n}^{\mathrm{o}} 114$ : Historia Henrici. In uno volumine. A. LAwrence-Mathers, Manuscripts in Northumbria in the Eleventh and Twelfth Centuries, Woodbridge, 2003, p. 258. 
Mais Ælred ne se livre pas à une imitation servile. Son opuscule est un panégyrique dédié à un homme (Gautier Espec), une noblesse, un ordre monastique (les cisterciens) et une victoire. Il donne à son discours une ampleur singulière, l'insérant dans une description détaillée des interactions aristocratiques entre l'Écosse et le Yorkshire. Son public n'est pas le même que celui d'Henri de Huntingdon. Il écrit, comme le montre la diffusion manuscrite régionale de cette œuvre, d'abord pour les moines cisterciens, les chanoines augustins et les barons de Northumbrie et non pour les clercs et les grands de la cour royale d'Angleterre comme l'archidiacre de Huntingdon ${ }^{22}$. En outre, le texte dans son ensemble, est bien plus nuancé que celui de Henri de Huntingdon qui, adoptant un point de vue épiscopal et "anglo-centré », se contente de présenter les Écossais et leur roi comme des ennemis, tandis qu' Elred cherche à épargner David et ses chevaliers, à atténuer leur part de responsabilité dans le conflit ${ }^{23}$. Il est d'autant plus singulier qu'il ait composé un discours radical et qu'il l'ait placé dans la bouche de Gautier Espec. Ælred inscrit peut-être son travail dans une rivalité littéraire et mémorielle, mais il propose une interprétation à la fois complémentaire et originale de la campagne.

Le texte de Richard d'Hexham nous prouve plus encore qu'il n'y a pas simple reprise d'une construction rhétorique première. Richard rapporte les événements qui vont de 1135 à 1139 en considérant avant tout la destinée de sa communauté d'Hexham. Il consulta peut-être une édition de l'Historia Anglorum, mais qui s'arrêtait en 1135, trois ans avant la bataille de l'Étendard (il peut aussi s'agir de l'Historia regum de Siméon de Durham). Comme Ælred, Richard connaissait mieux le contexte que Henri de Huntingdon. Il écrivit son récit très peu de temps après la bataille, sans doute avant ou au même moment que Henri de Huntingdon - qui composa son discours vers 1140 - et bien avant Elred $^{24}$.

À la différence des auteurs précédents, le chanoine est fidèle aux codes d'une historiographie traditionnelle faite pour rappeler et édifier, non pour distraire et séduire : il écrit pour sa communauté, cherche à rapporter, scrupuleusement, les faits dans l'ordre ${ }^{25}$. Il ne veut pas composer de brillantes périodes rhétoriques : il mentionne, du reste, les propos de Toustain au style indirect. Suivant pas à pas les étapes de la campagne, il se borne à consigner les mots d'ordre lancés par l'archevêque d'York au début de la mobilisation.

La narration de Richard, originale comme le soulignait son éditeur Richard Howlett, est donc indépendante de celle de l'archidiacre de Huntingdon ${ }^{26}$. Il est, de même, exclu que ce dernier ait pu exploiter le récit de

22. E. FreEMAN, Narratives..., op. cit., p. 31-53.

23. A. GLIDDEN, «Aelred the Historian... », op. cit., p. 182.

24. A. Gransden, Historical Writing..., op. cit., p. 216.

25. Ibid., p. 217. N. F. PARTNER, Serious Entertainments : the Writing of History in TwelfthCentury England, Chicago, Londres, 1977.

26. Chronicles of the Reigns of Stephen..., op. cit., R. HowletT éd., t. 3, p. LVII. 
Richard, dont la chronique n'a guère circulé et dont on ne détecte nulle trace dans 1'Historia Anglorum 27.

Il est presque certain, en revanche, qu' Ælred se soit inspiré de Henri de Huntingdon ; il a peut-être aussi utilisé la chronique de Richard d'Hexham, car il entretenait des liens assez étroits avec sa communauté. Mais il disposait d'autres sources d'informations, puisqu'il connaissait personnellement nombre de protagonistes de l'affrontement ${ }^{28}$.

Ainsi la tonalité, le style, la construction même de ces trois discours diffèrent-ils. Ils ne s'insèrent pas dans le même projet historiographique, ne visent pas les mêmes publics, ne dérivent pas, substantiellement, les uns des autres. Il est donc remarquable que, malgré ces distinctions importantes, nous retrouvions l'évocation de thèmes de mobilisation comparables, proches les uns des autres pour peu que l'on s'attache à l'idéologie qui les sous-tend.

\section{Les discours et leurs thématiques}

Il n'est pas nécessaire de faire l'inventaire de l'ensemble des motifs dont sont composées les différentes harangues. John Bliese qui a établi cette grammaire de la rhétorique guerrière a déjà effectué cette enquête pour les oraisons prêtées à Raoul Novel et à Gautier Espec ${ }^{29}$. Il faut simplement insister sur les axes majeurs des discours.

Dans les trois allocutions, la guerre entreprise est présentée comme légitime. Elle est livrée pour la défense de la patrie anglaise et northumbrienne, pour la protection de l'Église. Toustain oppose la «cause scélérate » des troupes du roi d'Écosse à la cause «juste et très pieuse » des troupes fidèles à Étienne de Blois qui se battent pour la « défense de la sainte Église et de leur patrie » ${ }^{30}$. Pour Raoul Novel, les armes anglo-normandes sont les instruments de la divine volonté ${ }^{31}$. Gautier Espec se montre le plus soucieux de définir avec exactitude les critères qui fondent la juste cause. Il souligne la légitimité des droits d'Étienne sur le trône et la nécessité de combattre pour lui. Il définit cette guerre juste en rappelant que les combats menés visent à rétablir la paix, à protéger les biens et les personnes ${ }^{32}$. Dans ces conditions, l'aide divine est assurée, à condition toutefois de faire pénitence,

27. Ibid., p. LVI. Henri de Huntingdon, Historia..., D. Greenway éd., p. LXXXV-CVII.

28. J. BLIESE, « Ælred of Rievaulx's Rhetoric... », op. cit., p. 554-556.

29. ID., «Rhetoric and Morale... », op. cit., p. 201-206 ; ID., « Ælred of Rievaulx's Rhetoric... », op. cit., p. 543-556.

30. RichaRd D'HeXham, De gestis..., op. cit., p. 160 : «Ipsorum vero causa justa ac piissima erat, quippe pro Sanctæ Ecclesiæ ac suæ patriæ defensione periculo se objicebant. »

31. Henri de Huntingdon, Historia..., op. cit., L. X, chap. 7, p. 714 : «Quod iustissimum sue dispositionis arbitrium per manus uestras hodie perficiet Deus. »

32. Ælled DE Rievaulx, Relatio..., op. cit., p. 187-188. 
rappelle Toustain ${ }^{33}$. Pour Ælred, les cohortes célestes et le Christ lui-même prendront les armes aux côtés des combattants du Yorkshire ${ }^{34}$.

Le complément logique de cette faveur divine sur le plan temporel, ce sont les récompenses spirituelles. La lutte est considérée comme sainte par les autorités religieuses. Le trépas de ceux qui se sont lancés dans une telle entreprise ne doit donc pas être craint : ils seront sauvés. Toustain sousentend que la mort des combattants sera méritoire ${ }^{35}$. Raoul Novel le dit ouvertement ${ }^{36}$. Gautier Espec invite ses compagnons à consacrer «leurs mains dans le sang des pécheurs ${ }^{37}$. La lutte est une expiation ${ }^{38}$. Tous les discours concordent donc: il s'agit d'une guerre pénitentielle, et même sainte, au sens de guerre qui sanctifie, ipso facto, ceux qui y prennent part du bon côté et avec une intention droite ${ }^{39}$.

La dénonciation de l'adversaire est la contrepartie de cette noble cause : le bon camp n'existe que si son vis-à-vis est l'instrument du diable. L'ennemi est démasqué, déprécié. Cela est fréquent dans les harangues, mais la particularité des compositions oratoires de la bataille de l'Étendard est que les crimes insupportables dont les Écossais se seraient rendus coupables sont longuement décrits ${ }^{40}$. Il semble que l'expédition que le neveu du roi d'Écosse, Guillaume Fils Duncan, conduisit jusqu'à l'abbaye de Furness, en juin 1138, ait laissé de très mauvais souvenirs aux populations et que la rumeur se soit très vite répandue des exactions commises par les hommes de Galloway ${ }^{41}$.

La conclusion que tire Aelred de sa dénonciation est frappante. Il place dans la bouche de Gautier Espec une diatribe d'une rare violence :

33. Richard D'HeXham, De gestis..., op. cit., p. 160.

34. FlRed DE RiEvaulX, Relatio..., op. cit., p. 188-189.

35. Richard D'HeXHAM, De gestis..., op. cit., p. 160-161.

36. Henri de Huntingdon, Historia..., op. cit., L. X, chap. 8, p. 716.

37. ÆlRed De Rievaulx, Relatio..., op. cit., p. 188 : «Consecrate manus vestras in sanguine peccatorum. »

38. J. FLORI, «Les héros changés en saints... et les saints en héros. Sacralisation et béatification du guerrier dans l'épopée et les chroniques de la première croisade », PRIS-MA : bulletin de liaison de l'équipe de recherche sur la littérature d'imagination du Moyen Âge, t. 15, 2, 1999, p. 262.

39. Th. Deswarte, «La "Guerre sainte" en Occident: expression et signification », M. Aurell, Th. Deswarte dir., Famille, violence et christianisation au Moyen Âge. Mélanges offerts à Michel Rouche, Paris, 2005, p. 331-349 ; ID., «Entre historiographie et histoire : aux origines de la guerre sainte en Occident », D. BALOuP, Ph. JossERAND dir., Regards croisés sur la guerre sainte. Guerre, idéologie et religion dans l'espace méditerranéen latin (XI ${ }^{e}-X I I^{e}{ }^{s}$.), Toulouse, 2006, p. 67-90. J. FLORI, La Guerre sainte. La formation de l'idée de croisade dans l'Occident chrétien, Paris, 2009 (2001). D. S. BACHRACH, Religion and the Conduct of War, ca 300-1215, Woodbridge, 2003.

40. ÆlRed De RievaulX, Relatio..., op. cit., p. 187-188 ; Richard D’HeXHAM, De gestis regis Stephani..., op. cit., p. 160 ; Henri DE HunTINGdon, Historia..., op. cit., L. X, chap. 8, p. 714.

41. Richard D'HeXham, De gestis..., op. cit., p. 152-153, p. 156-157 ; ÆLRED DE RievaulX, Relatio..., op. cit., p. 187. 
Qu'êtes-vous en train de faire, ô hommes très puissants ? Vous ne luttez pas contre des hommes mais contre des bêtes qui ne font preuve d'aucune humanité ni d'aucune bonté ; que le ciel a en horreur, que la terre repousse, que les mers chargent d'imprécations, que les lumières de ce monde, elles-mêmes, maudissent; eux que la terre n'absorba pas, que le ciel ne foudroya pas, que les mers ne submergèrent pas à la seule condition que ceux-ci fussent sauvés par vos victoires, que ceux-là périssent par vos mains ${ }^{42}$.

Il y a dans cette harangue un appel transparent à l'exercice d'une justice immanente ${ }^{43}$. Il est frappant de constater que les adversaires sont retranchés de l'humanité : ils s'apparentent à des bêtes. Richard d'Hexham dit, pareillement, (sans cependant prêter cette parole à Toustain) que les Pictes sont des «hommes bestiaux » ${ }^{44}$. L'affirmation de soi et du groupe, au prix de la destruction de l'autre, passe par le rejet de l'identité de l'ennemi et l'assimilation de son individualité à l'animalité ${ }^{45}$.

À suivre les trois orateurs, la guerre oppose des «civilisés » à des hommes incultes, brutaux et cruels. John Gillingham et Matthew Strickland ont bien défini l'importance d'une telle distinction, lorsque les troupes anglonormandes étaient engagées sur les marges du royaume d'Angleterre ${ }^{46}$. Les chroniqueurs anglo-normands insistent sur ces différences ethniques et culturelles, stigmatisant le comportement des « hordes » celtiques du roi d'Écosse. Les différences s'exacerbent et les lignes de démarcation se multiplient. Le camp de la civilisation s'oppose à celui de la sauvagerie, le camp de l'ordre à celui du chaos, le camp de l'Église à celui des « crypto-païens » et le camp des hommes à celui des bêtes...

Il y a donc une grande similitude entre les thématiques majeures des discours, qui autorise une interprétation plus large du rôle des prises de paroles au cours de cette campagne.

42. ÆlRed De RIEVAulX, Relatio..., op. cit., p. 188 : «Quid agitis, o viri fortissimi ? Non adversus homines dimicatis sed adversus bestias, quibus nihil humanitatis, nihil inest pietatis ; quos cœlum horret, quos abominatur terra, quos execrantur maria, quos ipsa mundi lumina detestantur; quos non ob aliud terra non absorbuit, non fumilnavit cœlum, non maria submerserunt, nisi ut vestris victoriis servarentur, vestris manibus interirent. » Influence de Bernard de Clairvaux? BERnARD DE ClaIRVAuX, Éloge de la nouvelle chevalerie. Euvres complètes XXXI, J. LeClercQ et P.-Y. EMERY éd., Paris, L. II, p. 56. Catalogue de l'abbaye de Rievaulx..., op. cit., p. $152, \mathrm{n}^{\circ} 33$ : «Libri Bernardi [...] et exhotatio ejusdem ad milites templi... »

43. P. Rousset, «La croyance en la justice immanente à l'époque féodale », Le Moyen Âge, t. 54, 1948, p. 225-248.

44. Richard D'HeXham, De gestis..., op. cit., p. 157.

45. E. MORIN, L'homme et la mort; Paris, 1976 (1951), p. 80-82.

46. J. Gillingham, «Conquering the Barbarians: War and Chivalry in Twelfth-Century Britain and Ireland », dans ID., The English in the Twelfth Century. Imperialism, National Identity and Political Values, Woodbridge, 2000, p. 41-58; M. STRICKLAND, War and Chivalry. The Conduct and Perception of War in England and Normandy, 1066-1217, Cambridge, 1996, p. 183-203. 


\section{La campagne de « prédication politico-militaire »}

Si l'on considère les écrits de l'ensemble des auteurs qui ont détaillé la campagne et que l'on est attentif à la totalité des mentions de prises de paroles (même celles dont la teneur exacte n'est pas rapportée), notre vision de la campagne change ; nous percevons les ramifications d'une vaste mobilisation précédée d'une intense opération de communication. Il semble, à lire attentivement les chroniqueurs, que l'on soit confronté à une inflation de harangues. Il n'y aurait plus alors deux ou trois discours, mais beaucoup plus.

Tentons de retrouver le fil de cette campagne de prédication, dans sa réelle grandeur. L'ensemble des chroniqueurs rapportent que, face à l'offensive écossaise, les barons du Yorkshire et de Northumbrie, livrés à euxmêmes en l'absence du roi Étienne, incertains de la conduite à tenir, se rassemblent autour de l'archevêque d'York, Toustain, dont le prestige est grand parmi les nobles de ce comté pour avoir passé une bonne partie de sa vie, en exil, à lutter pour défendre l'indépendance de sa province ecclésiastique. Il insuffle aux barons l'esprit de résistance ${ }^{47}$.

Nous avons déjà présenté, en partie, la portée des propos tenus par l'archevêque le 27 juillet, que Richard d'Hexham nous livre. Ces paroles sont essentielles, car elles constituent le noyau dur des allocutions à venir : il faut se convertir, faire pénitence; les ravages des Écossais sont une épreuve envoyée par Dieu; la guerre entreprise pour défendre le pays et l'Église est juste ; la mort au combat n'est pas à craindre puisque l'intention des combattants est droite. Toustain s'engage, enfin, à mobiliser les paroisses et à envoyer prêtres, bannières et reliques, au devant de l'armée ${ }^{48}$. En un mot, l'archevêque d'York prêche une guerre sainte.

Après s'être équipés sur leurs terres, les chevaliers se retrouvent à York et, durant trois jours, se confessent, font pénitence, offrent des aumônes. Enfin, ils reçoivent l'absolution ${ }^{49}$.

Trop affaibli par la maladie, l'archevêque ne peut accompagner les troupes et délègue Raoul Novel. Des serments de vaincre ou mourir sont prêtés, dont la portée ne doit pas être négligée dans le contexte d'une guerre civile où les fidélités sont fragiles ${ }^{50}$. L'armée se met en mouvement. Les

47. Ællred De Rievaulx, Relatio..., op. cit., p. 182 ; Richard d’HeXham, De gestis..., op. cit., p. 160 ; HenRI De HunTingdon, Historia..., op. cit., L. X, chap. 7, p. 712 ; Jean D’Hexham, Symeonis historia..., op. cit., p. 292 ; D. Nicholl, Thurstan, Archbishop of York (1114-1140), York, 1964, p. 215-233.

48. RichaRd D'HeXham, De gestis..., op. cit., p. 161.

49. Richard D'HeXham, De gestis..., op. cit., p. 161 ; Ælled De Rievaulx, Relatio..., op. cit., p. 189.

50. A. SQuire, Aelred of Rievaulx..., op. cit., p. 80-81 ; ÆLlRED DE RIEvaulX, Relatio..., op. cit., p. 189 ; Richard D'HeXham, De gestis..., op. cit., p. 162. JeAn DE WorCester, Chronicon..., op. cit., p. 252. 
prêtres accompagnent les troupes, prêchant et veillant à la qualité de la vie spirituelle des combattants ${ }^{51}$.

Le jour même de la bataille, Gautier Espec, d'après Ælred de Rievaulx, n'est pas le seul à prendre la parole : l'abbé cistercien mentionne aussi le rôle de l'évêque des Orcades. C'est lui, comme chez Henri de Huntingdon, qui donne l'absolution aux troupes avant le combat et qui les encourage par son prêche qu' $Æ l$ red ne fait que mentionner ${ }^{52}$. Un manuscrit $\mathrm{du} \mathrm{XV}^{\mathrm{e}}$ siècle insère cependant à cet endroit, dans la Relatio de Standardo, le discours composé par Henri de Huntingdon pour Raoul Novel ${ }^{53}$ ! Ælred de Rievaulx s'efforce, sans doute, de faire coïncider sa version des faits avec celle de son prédécesseur, tout en lui donnant une plus vaste dimension. Mais il veut aussi rendre compte de l'ampleur de la mobilisation qui a précédé la bataille. Du reste, l'abbé de Rievaulx n'ajoute pas seulement au discours de Gautier celui de Raoul Novel, il évoque aussi les nombreux sermons militaires prêchés en ce jour : les prêtres exhortent les troupes avant la bataille, relayant probablement la parole de leurs supérieurs ${ }^{54}$.

Pour bien saisir l'importance de l'effort de propagande entrepris par Toustain, il faut s'arrêter quelques instants sur les règles qui président à la composition des discours militaires au XII ${ }^{\mathrm{e}}$ siècle. La bataille devient la clef de voûte de la narration. Les épisodes essentiels sont aimantés par elle. Les «nouvelles tendances» stylistiques du récit historique incitent Henri de Huntingdon ou Ælred de Rievaulx à resserrer les nœuds de l'intrigue, à concentrer l'action, à placer juste avant le combat les paroles qui ont servi à galvaniser les troupes, de façon à faire ressortir l'intensité de la situation. Mais ces compositions ne prennent vraiment sens qu'associées aux autres témoignages. Assurément, la préparation au combat, la prédication politique, militaire et religieuse, est partie de beaucoup plus loin.

Les discours se sont probablement échelonnés sur plusieurs jours, surtout si l'on considère que l'allocution initiale de Toustain à York a eu lieu le 27 juillet, soit plus d'un mois après la défaite de Cliterhoe (10 juin) et plus de trois semaines avant la bataille de l'Étendard (22 août). Entre-temps, l'armée anglaise s'est concentrée dans la forteresse de Thirsk, attendant les Écossais et leur barrant la route du sud ${ }^{55}$. C'est là que Raoul Novel et ses clercs auraient rejoint l'armée, chargés par l'archevêque Toustain de veiller

51. Jean d'Hexham, Symeonis historia..., op. cit., chap. 6, p. 293 ; Richard D’Hexham, De gestis..., op. cit., p. 162. JEAN DE WORCESTER, Chronicon..., op. cit., p. 252.

52. ÆLRED DE RIEVAUlX, Relatio..., op. cit., p. 195.

53. Ibid., p. 195 , note 5 .

54. Ibid., p. 192. Sur les liens entre sermons et discours voir C. GIRBEA, « Discours persuasifs et conscience religieuse dans les romans arthuriens (XII ${ }^{\mathrm{e}}-\mathrm{XII}^{\mathrm{e}} \mathrm{s}$.) », dans M. AURELL dir., Convaincre et persuader..., op. cit., p. 153-189.

55. Richard D'Hexham, De gestis..., op. cit., p. 161 ; Jean D'Hexham, Symeonis historia..., op. cit., chap. 5, p. 293. 
sur le moral et la vie spirituelle des combattants ${ }^{56}$. Cette période d'incertitude et de vigilance armée était propice aux ultimes tentatives de négociations, mais surtout aux exhortations stimulantes.

\section{Les ressorts de la mobilisation}

\section{Intérêts, choix politiques et idéologie}

Les différents discours - qu'ils soient « rapportés » ou mentionnés sont les traces d'une prédication qui eut, semble-t-il, un rôle considérable dans la victoire anglaise. Tous les orateurs et meneurs cités se connaissaient et partageaient les mêmes intérêts. Tous ont choisi d'adhérer à la cause du roi Étienne (malgré les liens qu'ils entretenaient aussi avec le roi d'Écosse) et de participer activement à la diffusion d'idées favorables à la cause du roi d'Angleterre.

À l'origine de cette mobilisation, le rôle de l'archevêque Toustain est fondamental. Il s'est montré, dès le début, fidèle à Étienne de Blois. Et cela peut s'expliquer : alors qu'il était en exil en France, il fut reçu chez la mère d'Étienne, Adèle, fille de Guillaume le Conquérant, avec laquelle se noue une profonde affection. Il n'est pas présent au couronnement d'Étienne mais apparaît à sa cour dès Pâques 1136. En 1137, il négocie une trêve avec David à Roxburgh. En présidant un concile à Northampton le 10 avril 1138 (le siège de Cantorbéry est alors vacant), il favorise la cause d'Étienne de Blois ${ }^{57}$. Il est même possible que celui-ci lui ait confié le pouvoir pour le nord du royaume. Selon Richard d'Hexham. Toustain y exerce son autorité à la fois en tant qu'archevêque et en tant que représentant royal ${ }^{58}$. Raoul Novel, évêque des Orcades, est son double, sa voix auprès de l'armée durant la campagne.

Gautier Espec a été, avec Eustache Fils Jean, durant la seconde moitié du règne d'Henri $\mathrm{I}^{\text {er }}$, un des hommes de confiance du roi pour le nord de l'Angleterre, un des ces hommes nouveaux promus par le service du souverain ${ }^{59}$. Certes Gautier Espec connaissait bien le roi d'Écosse qui avait favorisé la création de Rievaulx mais leurs liens ne semblent, en fin de compte, pas si forts : il n'était pas, contrairement à d'autres, vassal de David ${ }^{60}$ et

56. Richard D'HeXham, De gestis..., op. cit., p. 162.

57. D. Nicholl, Thurstan..., op. cit., p. 221-224.

58. Richard D'HeXham, De gestis..., op. cit., p. 160 : «Unde et ex divina auctoritate, quæ ei commissa fuerat, et ex regia potestate, quæ illi tunc in hoc negotio tradita erat... »

59. Cinq actes du roi Henri I ${ }^{\mathrm{er}}$ lui sont adressés : Early Yorkshire Charters Being a Collection of Documents Anterior to the Thirteenth Century Made from the Public Records, Monastic Chartularies, Roger Dodsworth's Manuscripts and other Available Sources, éd. W. Farrer, Edinburg, 1916 : t. $1, n^{\circ} 98$, p. $92-93$, no 500 , p. $385 ;$ t. 2 , no 935 , p. 274, n $^{\circ} 1163$, p. 454 , no 866 , p. 211. Il est témoin d'actes d'Henri $\mathrm{I}^{\mathrm{er}}$ à six reprises : Ibid., t. $1, \mathrm{n}^{\circ} 132, \mathrm{p} .120-121, \mathrm{n}^{\circ} 429$, p. $336, n^{\circ} 449$, p. 348 ; t. 2 , no 806 , p. 151 ; t. 3 , no 1428 , p. $129-133$, no 1435 , p. 141-142.

60. R. L. L. Graeme, The Normans..., op. cit., p. 146-147. 
n'apparaît pas dans ses chartes ${ }^{61}$. Ses intérêts le portaient donc plus à demeurer fidèle au roi d'Angleterre, qu'il soutient très vite. Il est témoin d'une charte du roi à Westminster dès la fête de Pâques $1136^{62}$. L'un de ses neveux, Jordan de Bussey, se distingue par une résistance héroïque face aux armées écossaises en défendant, pour lui, en janvier 1138, le château de Carham ${ }^{63}$.

Il n'est pas impossible que Gautier Espec ait pu haranguer brillamment l'armée lors de la rencontre de l'Étendard. Du moins était-ce, à l'époque, crédible. Ælred le décrit comme un personnage charismatique, exerçant une autorité morale, un véritable ascendant sur les nobles anglo-normands : c'est un homme vieux et sage, passionné d'histoire ${ }^{64}$. Ces derniers faits sont confirmés par Geoffroy Gaimar qui aurait utilisé pour son Estoire un livre en latin traduit du gallois, emprunté par Gautier à Robert de Gloucester ${ }^{65}$.

Gautier de Gant, très âgé au moment des faits, prit également la parole pour encourager les barons normands au début de la campagne : il semble avoir joué un rôle notable dans la mobilisation de la noblesse. Sa fidélité à Étienne de Blois est assez assurée : son fils, combattant auprès du roi, fut fait prisonnier à la bataille de Lincoln ${ }^{66}$.

Gautier Espec, Toustain et Gautier de Gant ont enfin collaboré au renouveau de la vie religieuse dans le nord de l'Angleterre et, plus précisément, à l'implantation des chanoines augustins puis des cisterciens ${ }^{67}$. Toustain fonda l'abbaye cistercienne de Fontaines. Gautier installa des chanoines augustins à Kirkham (1122) et les cisterciens à Rievaulx (1132) et à Wardon (1135). Toustain ne peut avoir ignoré les négociations menées entre Bernard de Clairvaux, Henri Ir $^{\text {r }}$ et Gautier Espec, puisqu'il approuva la fondation de Rievaulx ${ }^{68}$. Gautier de Gant fonda le prieuré de chanoines augustins de Bridlington vers 1113 et c'était un des principaux et des premiers donateurs de l'abbaye de Rievaulx. Son fils Gilbert poursuivit son action en créant en 1146 ou 1148 une abbaye cistercienne à Rufford, dont il fit une fille de Rievaulx ${ }^{69}$. Étienne de Blois confirma, une à une, ces créations et ces dona-

61. The Charters of King David. The Written Acts of David I King of Scots 1124-1153 and his Son Henry Earl of Northumberland, 1139-1152, G.W.S. BARROW éd., Rochester, 1999. Eustache Fils Jean apparaît, lui, à plusieurs reprises : no 73, p. 88 ; no 82, p. 92-93; n 106, p. 105.

62. Early Yorkshire Charters..., op. cit., t. 2, no 867, p. 214 (1136).

63. D. NichOll, Thurstan..., op. cit., p. 213-233.

64. Ælred DE Rievaulx, Relatio..., op. cit., p. 185.

65. Geoffroy Gaimar, L'Estoire des Engleis, A. Bell éd., Oxford, 1960, p. 225 vers 6448-6458. Voir I. SHORT, « Gaimar's Epilogue and Geoffrey of Monmouth's Liber vetustissimus », Speculum, t. 69, 1994, p. 323-343.

66. Ælned de Rievaulx, Relatio..., op. cit., p. 182. JeAn D'Hexham, Symeonis historia..., op. cit., chap. 13, p. 303.

67. D. BAKER, «Patronage in the Early Twelfth-Century Church : Walter Espec, Kirkham and Rievaulx », Traditio-Krisis-Renovatio aus theologischer Sicht, B. JASPERT éd., Marburg, 1976, p. 92-100.

68. A. SQUiRe, Aelred of Rievaulx..., op. cit., p. 17-18.

69. E. JAMROZIAK, Rievaulx Abbey and its Social Context, 1132-1300. Memory, Locality and Networks, Turnhout, 2005, p. 81-83. 
tions $^{70}$. Certes le roi d'Écosse est loin d'être absent de ce mouvement de renouveau religieux ${ }^{71}$. Mais dans ses Genealogia regum Anglorum et dans son Eulogium Davidis regis Scottorum, le seul reproche que lui fait Ælred est sa campagne de 1138, car ses troupes risquaient de remettre en cause, par leurs déprédations, l'entreprise de reconquête religieuse ${ }^{72}$.

C'est en grande partie un petit noyau d'hommes influents d'où émergent ces personnalités (auxquelles on pourrait ajouter Robert de Bailleul, dont l'action fut décisive lorsqu'il amena les renforts royaux à York en juillet ${ }^{73}$ ), qui parvint à convaincre une noblesse hésitante, ayant parfois tissé des liens forts avec David, de se rallier à Étienne ${ }^{74}$.

Pourtant, la situation de David d'Écosse semblait, au départ, très favorable. Depuis la bataille de Cliterhoe (10 juin 1138), les chevaliers du nord de l'Angleterre étaient démoralisés ou tentés de passer du côté de David ${ }^{75}$. Cela leur éviterait de voir leurs terres ravagées et leur assurerait les faveurs et la protection d'un souverain très proche, qui avait encouragé le développement de la «civilisation normande » en Écosse ${ }^{76}$. D'ailleurs Jean d'Hexham écrit que, dès février 1138, Étienne de Blois qui s'apprêtait à poursuivre David à Roxburgh renonça, craignant des trahisons dans son propre camp ${ }^{77}$. C'est à cette occasion que, soupçonneux, il enleva à Eustache Fils Jean le château de Bamburgh, ce dernier se ralliant alors à Mathilde et donc au roi d'Écosse. Robert Bruce semble hésiter longtemps avant de choisir le camp d'Étienne de Blois. L'un de ses fils, Adam, l'héritier des terres anglaises, se trouvait avec son père, tandis que l'autre, Robert, héritier des terres écossaises de la famille à Annandale, combattait du côté de David ${ }^{78}$. Durant l'été 1138, la situation était donc critique pour la noblesse du Yorkshire, indécise, prise entre deux fidélités, entre un souverain écossais proche et menaçant et un souverain anglais absent, dont la légitimité commençait à être remise en cause. Elle aurait pu facilement basculer dans le camp de David et se rallier à ses rêves d'une grande Northumbrie, dépendante de l'Écosse.

70. Par exemple confirmation de la fondation de Rievaulx et de donations faites à Fontaines: Regesta regum Anglo-Normannorum 1066-1154, t. 3, Regesta regis Stephani ac Mathildis Imperatricis ac Gaufidi et Henrici Ducum Normannorum, 1135-1154, H. A. CRONNE, R. H. C. DAvis in continuation of the work of the late H. W. C. DAvis éd., Oxford, 1968, n 335 , p. 126-127, no 716 , p. 263.

71. The Charters of King David..., op. cit., no 120, p. 110-111 ; n $\mathrm{n}^{\mathrm{o}} 121$, p. 111-112.

72. A. SQuire, Aelred of Rievaulx..., op. cit., p. 83 ; ÆLRED DE RIEVAUlX, Eulogium Davidis Regis Scottorum, Pinkerton's Lives of the Scottish Saints, W. M. METCALFE éd. revue, Paisley, 1889, t. II, chap. 6, p. 275.

73. Richard D'HeXham, De gestis..., op. cit., p. 161 ; JeAn D'HeXham, Symeonis historia..., op. cit., chap. 5 , p. 292.

74. D. NiCHOLL, Thurstan..., op. cit., p. 222.

75. JEAN D'HeXHAm, Symeonis historia..., op. cit., chap. 5, p. 291.

76. R. R. DAvies, Dominion and Conquest. The Experience of Ireland, Scotland and Wales, 1100-1300, Cambridge, 1990, p. 51, p. 114.

77. Ibid., chap. 4, p. 291.

78. A. SQuire, Aelred of Rievaulx..., op. cit., p. 80. R. M. Blakely, The Brus Family in England and Scotland, 1100-1295, Woodbridge, 2005, p. 28-30. 
Les axes majeurs de la campagne de prédication politico-militaire menée tambour battant, à l'initiative de Toustain et des nobles mentionnés plus haut, sont bien choisis. Le ralliement à Étienne de Blois est justifié par la fidélité au roi comme garant de l'ordre et de la paix du royaume d'Angleterre face à l'agression. Ce ralliement se confond avec la cause de l'Église, dont les prêtres et les autels ont souffert de l'avancée écossaise.

La mobilisation s'effectue donc sur des thèmes consensuels de défense du royaume, des populations et de la foi, sans que jamais l'on ne s'en prenne directement au roi d'Écosse. Sont en revanche exploitées les fautes politiques commises par David alors qu'il cherche à étendre sa souveraineté sur le Yorkshire, au-delà de son aire de domination coutumière en s'appuyant plus sur les hommes de Galloway que sur ses chevaliers anglonormands ${ }^{79}$. La lutte peut dès lors s'organiser contre les barbares qui risquent de renverser la civilisation et l'œuvre de reconquête monastique entreprise dans le nord de l'Angleterre. L'accent est placé volontairement sur les horreurs dont se rendent coupables les Écossais ${ }^{80}$. La figure inquiétante du « Picte », de l' « homme de Galloway », sert à masquer les divisions internes à l'aristocratie normande.

Ainsi l'échec de David est-il surtout le fruit d'une incapacité à obtenir l'appui du groupe chevaleresque du Yorkshire et de la Bernicie. La propagande politico-militaire dont les arguments sont formalisés de façon rhétorique dans les harangues prend ici tout son sens. Toustain, Raoul Novel, Gautier Espec, Gautier de Gant et leurs fidèles, ont agi pour la cause d'Étienne et celle de «leur » comté en prétendant ne faire que combattre l'envahisseur septentrional : les barbares. En «instrumentalisant» la peur qu'inspiraient les hommes de Galloway, ils profitaient aussi des tensions internes à l'armée écossaise composée d'un petit groupe de féodaux francs, jalousés par les Scots et les Pictes ${ }^{81}$. Au contraire les troupes anglaises et les chevaliers normands se trouvaient réunis face au danger dans la défense de ce qui était désormais leur patrie commune. En célébrant les exploits des fils de Rollon, Raoul Novel et Gautier Espec exprimaient aussi la résurgence du peuple anglais qui participait à la bataille et dont les nobles, d'origine continentale, se considéraient comme les chefs naturels ${ }^{82}$.

79. A. LAWrenCe-Mathers, Manuscripts..., op. cit., p. 250-251.

80. Ælred De Rievaulx, Relatio..., op. cit., p. 187-188 ; Henri DE Huntingdon, Historia..., op. cit., L. X, chap. 8, p. 714 et 716; RICHARD D'HEXHAM, De gestis..., op. cit., p. 152, 154 et 156 ; Jean D’Hexham, Symeonis Historia..., op. cit., chap. 4, p. 290 ; J. Gillingham, «Conquering the Barbarians... », op. cit., p. 67-84.

81. M. STRICKLAND, « Securing the North : Invasion and the Strategy of Defence in TwelfthCentury Anglo-Scottish Warfare », dans ID. éd., Anglo-Norman Warafre : Studies in Late AngloSaxon and Anglo-Norman Military Organization and Warfare, Woodbridge, 1992, p. 223-224.

82. J. Gillingham, « Henry of Huntingdon and the Twelfth-Century Revival of the English Nation », dans ID. éd., The English in the Twelfth Century. Imperialism, National Identity and Political Values, Woodbridge, 2000, p. 129-130. 
En faisant preuve d'une grande habileté dans le choix des formules mobilisatrices, Toustain et les siens se gardaient bien d'aborder trop directement la question épineuse de la légitimité d'Étienne de Blois (seul Ælred y fait référence en reprenant peut-être le préambule de la charte d'Oxford sur les libertés de l'Église ${ }^{83}$ ). Discuté, dès le printemps 1138, dans l'entourage de Robert de Gloucester, le sujet empoisonné des serments de fidélité n'est pas mentionné ${ }^{84}$. Les partisans d'Étienne préférèrent mettre adroitement en avant la nécessité de défendre la patrie et l'Église. C'est cet aspect qui perdure dans l'image de l'événement que nous conservent les harangues militaires, qui procèdent à une simplification et à une cristallisation radicales des enjeux de la campagne.

La confrontation de l'Étendard indique que l'usage public de la parole, sous quelque forme que ce fût, était indispensable au pouvoir des princes, plus que jamais en temps de guerre lorsque l'essentiel était de s'assurer le concours de la noblesse dans des circonstances déterminantes. Mais la réussite de l'entreprise de Toustain est aussi fille de sa capacité à mettre en mouvement une partie de la population anglaise (les humbles paroissiens) associée à cette lutte.

\section{La mobilisation au-delà du cercle aristocratique}

Dès le départ de la résistance orchestrée par Toustain, il est question de lever des troupes au sein des paroisses: les hommes valides vont au combat sous la direction de leurs prêtres. Seule l'Église du Yorkshire, par l'intermédiaire du clergé, pouvait appeler la population à se dresser contre l'envahisseur. D'ailleurs les pénitences prescrites avant le départ de l'expédition ne sont pas réservées aux seuls nobles. Selon Richard d'Hexham, tout le peuple y participe et reçoit l'absolution et la bénédiction de Toustain à York ${ }^{85}$. De même, Ælred de Rievaulx dit bien que, juste avant la bataille, des prêtres vêtus de leurs habits sacrés, portant les croix et les reliques des saints, parcourent les rangs, réconfortant le peuple par des sermons et des prières ${ }^{86}$.

Cela implique que les mots d'ordre de Toustain d'York aient été relayés, en langue vernaculaire, dans les paroisses et au sein de l'armée. Le discours que Henri de Huntingdon fait prononcer à Raoul Novel est significatif puisque ce dernier commence par s'adresser aux nobles et aux grands

83. Regesta regum Anglo-Normannorum..., op. cit., tome 3, no 271, p. 96 ; ÆLRED DE Rievaulx, Relatio..., op. cit., p. 187.

84. D. CROUCH, The Reign..., op. cit., p. 123-124.

85. RichaRd D'HeXham, De gestis..., op. cit., p. 161 : «Itaque post acceptam privatam pœnitentiam, illis pariter et omni populo archipræsul triduanum cum eleemosinis indixit jejunium, ac deinde absolutionem, et benedictionem Dei et suam eis sollempniter tribuit. »

86. ÆLRED De RievaulX, Relatio..., op. cit., p. 192 : « [...] sacerdotes sacris vestibus candidati, cum crucibus et reliquiis Sanctorum exercitum ambiebant, et sermone simul et oratione populum decentissime roborabant. » 
pour finalement donner sa bénédiction à toute la foule qui répond, en une immense clameur: «Amen! Amen! ${ }^{87}$.

En outre les signes visibles par tous qui accompagnent ces prises de paroles constituent, en eux-mêmes, une forme de discours : la présence des reliques et les sacrements que dispensent les membres du clergé rassurent et réconfortent l'ensemble des combattants. Cela obéit à une mise en scène, avec l'élévation d'une construction qui servit de point de ralliement et donna son nom à la bataille ${ }^{88}$. Il s'agissait d'un mât fixé sur une structure en bois, une sorte de chariot assimilable au carroccio italien que les communes emmenaient à la bataille et sur lequel se trouvaient les enseignes de la cité ainsi qu'un autel servi par des prêtres ${ }^{89} \ldots$

Sur le mât avaient été accrochés le saint sacrement, la bannière royale et les bannières des saints de plusieurs sièges épiscopaux : Pierre pour York, Cuthbert pour Durham, Wilfrid pour Ripon et Jean pour Beverley ${ }^{90}$. C'est derrière ces saints que les humbles du Yorkshire se pressent pour contrer l'attaque écossaise. Ils symbolisent les différentes «patries » : céleste, royale, locale. Ces emblèmes se conjuguent pour renforcer le courage de l'armée ${ }^{91}$. Symboliquement, le Christ, par la présence réelle du saint sacrement qui pallie l'absence d'Étienne de Blois, en est le chef ${ }^{92}$.

Les textes qui se rapportent à la bataille de l'Étendard mentionnent, de façon assez concordante, un ensemble de prises de paroles qui nous permettent de comprendre les modalités de la diffusion d'un message politique en contexte militaire. Les trois harangues ne sont pas que des créations littéraires, détachées du réel. Prises dans leur ensemble, elles reflètent les thématiques partisanes qui furent à même de galvaniser les énergies des hommes du Yorkshire.

La victoire de l'Étendard manifeste l'incapacité du roi David d'Écosse à se rallier la noblesse anglo-normande au-delà des rivières Tyne et Tees. Cette difficulté s'explique par le succès exemplaire d'une opération de propagande menée par l'archevêque Toustain et ses proches. La réussite est d'autant plus impressionnante que cette victoire éclatante, bien que non exploitée, fut autant l'expression de la fidélité envers Étienne de Blois que l'événement

87. Henri de Huntingdon, Historia..., op. cit., L. X, chap. 8, p. 714 : « Proceres Anglie, clarissimi Normannigene [...]»; L. X, chap. 9, p. 716 : «Respondit omnis populus Anglorum, et resonuerunt montes et colles, "Amen, Amen" ». Sur l'idée d'une communauté politique se définissant des compagnons du roi au reste de la population: F. FORONDA, « La propagande monarchique... », dans M. AuRell dir., Convaincre et persuader.., op. cit., p. 287-288.

88. ÆLRED De RievaulX, Relatio..., op. cit., p. 189.

89. A. GlidDEn, «Aelred the Historian...», op. cit., p. 176 ; D. Nicholl, Thurstan..., op. cit., p. 227, note 45. Je n'ai malheureusement pas pu consulter : H. ZUG TUCCI, « Il carrocio nella vita communae italiana », Quellen und Forschungen aus italiensichen Archiven und Bibliotheken, t. 65, 1985, p. 1-104 et E. Voltmer, Il Carrocio, Turin, 1994.

90. Richard D'HeXham, De gestis..., op. cit., p. 163 ; E. Freeman, Narratives of a New Order. Cistercian Historical Writing in England, 1150-1220, Turnhout, 2002, p. 46.

91. Ælled de RievaulX, Relatio..., op. cit., p. 184-185; D. S. Bachrach, Religion and the Conduct of War, ca 300-1215, Woodbridge, 2003, p. 153-161.

92. RichaRd D'HeXham, De gestis..., op. cit., p. 163. 
fondateur à l'origine d'engagements aristocratiques durables à ses côtés. En ce cas précis, au terme d'une efficace mobilisation, sa légitimité ne semblait pas contestable : le destin du roi, bien qu'absent, s'était confondu avec celui de sa noblesse, de son Église et de ses sujets du nord de l'Angleterre.

Xavier Storelli, 17 rue Maryse Bastié, 86580 Biard

\section{Les harangues de la bataille de l'Étendard (1138)}

Deux harangues ont été composées pour la bataille de l'Étendard (22 août 1138). Elles sont attribuées par Henri de Huntingdon, à Raoul Novel évêque des Orcades et, par Ælred de Rievaulx, à Gautier Espec, seigneur de Helmsley. Il faut absolument y ajouter les propos de Toustain, archevêque d'York, que rapporte Richard d'Hexham. Même s'il serait absurde d'y rechercher les retranscriptions exactes de paroles qui auraient été, effectivement, prononcées telles quelles, la voix des orateurs de chair paraît, pour une fois dans ce sousgenre historiographique des oraisons guerrières, imprégner celle des orateurs de papier.

Mis en parallèle et associés au témoignage d'autres chroniques, ces discours nous autorisent à appréhender la mise en œuvre d'une prédication politicomilitaire d'assez vaste ampleur et à large destination, puisqu'elle permit la mobilisation des nobles et des contingents plus populaires des paroisses. C'est toute une campagne de propagande qui se révèle, si l'on considère l'ensemble des mentions de prises de paroles. Elle joua un rôle déterminant dans la victoire anglo-normande finale, en masquant les tensions internes au groupe aristocratique anglo-normand et en présentant le camp écossais comme celui de la sauvagerie face au camp anglais de l'ordre et de l'Église.

Bataille de l'Étendard - harangue - propagande - historiographie - chevalerie

\section{The harangues of the Battle of the Standard (1138)}

Henry of Huntingdon and Ælred of Rievaulx wrote that, before the Battle of the Standard (August 1138) began, respectively Walter Espec, lord of Helmsley, and Ralph Novel, bishop of the Orkneys, exhorted the Anglo-Norman army ; and Richard of Hexham mentioned the speech Thurstan, archbishop of York, delivered in July, a few weeks before the battle. Of course, these orations are not verbatim reports of harangues as they were actually given, but these ones are nevertheless particular, as they are profoundly linked to the military and political background.

Studying closely these battle orations and allusions to speeches, we can discover a real propaganda campaign, which was decisive in the English victory of the Standard. The mobilization of knights and of contingents sent by villages, each headed by their priest, was possible. In these orations, Galwegians'savagery is denounced, whereas the Anglo-Norman army is said to fight for the Church and the peace. At that moment, this kind of arguments was the best way to prompt nobles and people to fight for King Stephen against King David, in the north of England.

Battle of the Standard - oration - propaganda - historiography - knighthood 